\title{
A COST 240 Benchmark Test for Beam Propagation Methods Applied to an Electrooptical Modulator Based on Surface Plasmons
}

\author{
H. J. W. M. Hoekstra, P. V. Lambeck, G. J. M. Krijnen, J. Ctyroky, M. De Minicis, \\ C. Sibilia, O. Conradi, S. Helfert, and R. Pregla, Senior Member, IEEE
}

\begin{abstract}
Modeling of a waveguide polymer electrooptic (EO) modulator based on a resonant excitation of surface plasmons was used as a benchmark test for several beam propagation methods (BPM's). Wave-optical analysis of the structure is presented, and the results of four implementations of three numerical modeling methods are mutually compared and discussed.
\end{abstract}

Index Terms - Beam propagation method (BPM), benchmark test, electrooptic (EO) modulator, modeling, surface plasmons, waveguides.

\section{INTRODUCTION AND PROBLEM Definition}

$\mathbf{S}$ EVERAL benchmark tests for beam propagation methods (BPM's) have been formulated and performed recently within the framework of COST 240 Project [1]-[3]. These tests studied in some details the behavior of different BPM methods in various situations: the influence of a step-index waveguide tilt was tested in [1], low- and high-contrast waveguide tapers in [2], and waveguides with simultaneous loss and gain with strongly bent field phase fronts in [3]. In the present benchmark test, the BPM methods are applied to high-contrast waveguides containing thin $(70 \mathrm{~nm})$ lossy metallic layers supporting surface plasmons.

Surface plasmons (SP's) are transverse magnetic (TM)-type guided-lightwaves propagating along a boundary between two materials. Assuming, for the moment, nonabsorbing materials, the condition for their existence is that the dielectric constant of one of those materials, say $a$ is negative, while $\left|\varepsilon_{a}\right|>\varepsilon_{b}$. For visible and near infrared (IR) light that condition is obeyed by metals like silver, gold, and aluminum.

For the simplest SP supporting structure, a stratified layer structure consisting of two semi-infinite layers only, analytical expressions can be derived for the values of the propagation constant $\beta$ and the penetration depth $d_{p} \rightarrow(1 / e$-value $)$ of the

Manuscript received March 16, 1998.

H. J. W. M. Hoekstra, P. V. Lambeck, and G. J. M. Krijnen are with the MESA Research Institute, University of Twente, 7500 AE Enschede, The Netherlands.

J. Ctyroky is with IREE, Chaberska 57, Prague 818251 Czech Republic.

M. De Minicis and C. Sibilia are with the Universita di Roma "La Sapienza," Rome 00161 Italy.

O. Conradi, S. Helfert, and R. Pregla are with the Fern Universität Hagen, Hagen D-58084 Germany.

Publisher Item Identifier S 0733-8724(98)07400-3.
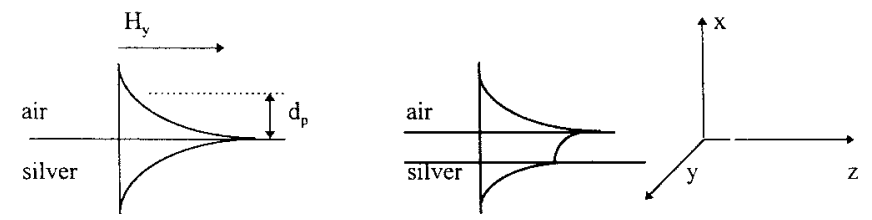

Fig. 1. Field profiles of surface plasmons.

field, exponentially decaying into the dielectric medium

$$
\begin{aligned}
\beta & =k_{0} \sqrt{\frac{\varepsilon_{a} \varepsilon_{b}}{\varepsilon_{a}+\varepsilon_{b}}} \\
d_{p} & =\operatorname{Re}\left\{1 / \sqrt{\beta^{2}-k_{0}^{2} \varepsilon_{b}}\right\}=\operatorname{Re}\left\{\sqrt{-\left(\varepsilon_{a}+\varepsilon_{b}\right) / \varepsilon_{b}^{2}}\right\} / k_{0}
\end{aligned}
$$

where $k_{0}$ is the wavenumber in vacuum, $2 \pi / \lambda$. Because the metal layer is strongly absorptive, it follows from the relation (1a) that $\beta$ is a complex quantity:

$$
\beta=\beta^{\prime}+i \beta^{\prime \prime}
$$

where $\beta^{\prime}$ determines the propagation velocity of the SP and $\beta^{\prime \prime}$ its attenuation, and hence its decay length into the propagation direction. For the system given in Fig. 1 (left), at $\lambda=633$ $\mathrm{nm}, \beta=(10.2545+i 0.0102) \mu^{-1}$, and $d_{p}=390 \mathrm{~nm}$ can be calculated.

In case that the layer-stack consists of more layers, numerical methods generally based on the transfer matrix method are required for calculating the propagation constant and the field profile.

The large field concentration near the metal surface, making the propagation constant very sensitive to small changes in the refractive index of thin layers on top of the silver layer (see Fig. 1) is exploited in (bio)-chemical sensors, where the index changes are (bio)-chemically induced.

Surface plasmons are generally excited by prism coupling or grating coupling, the $\beta$-value can be deduced from the excitation conditions, typically the angle of incidence of the exciting laser beam.

Another way of excitation, that recently became popular is resonant tunneling of power from a guided mode propagating in a well-defined three-layer waveguide (see Fig. 2) [4]. 


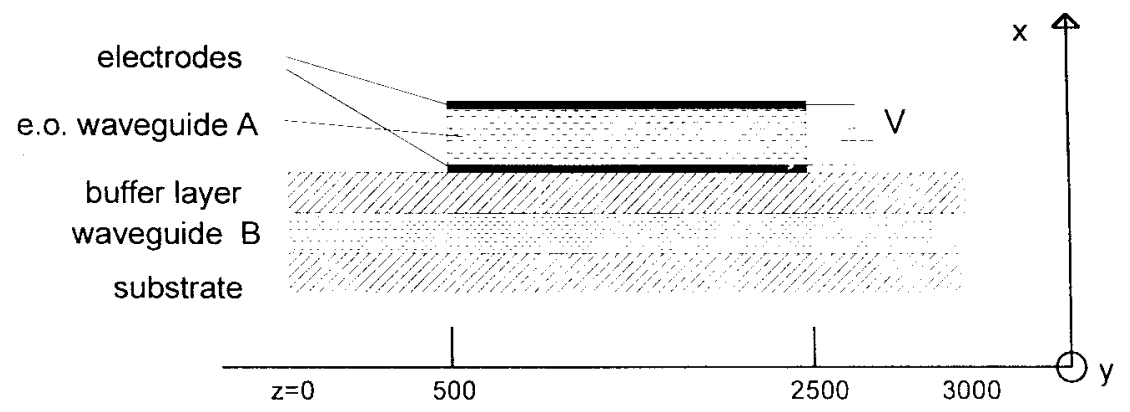

Fig. 2. The waveguide structure used in the benchmark test. Details are given in Table I.

The tunneling condition requires that the difference between the propagation constants of the waveguide mode and the SP is not too large compared to the coupling constant. In sensing applications, the refractive index of the (thin) layer adjacent to the metal layer can be found, e.g., by varying the wavelength of the guided mode, and determining the wavelength for which light intensity has a minimum at the waveguide exit. At this resonant wavelength, the tunneling into the SP is the strongest, and most power is absorbed in the metal. However, resonance condition can also be attained by index changes: by launching a TM mode of a given wavelength into the waveguide, and deliberately controlling the refractive index of the dielectric, the waveguiding system can be switched from a transmission state to an extinction state: an intensity modulator is within reach.

In the optical waveguide structure of Fig. 2, the well controlled refractive index changes are achieved by varying the electric field within a thin layer consisting of an electrooptical (EO) material sandwiched between two silver layers. These silver layers function both as electrodes and as SP-supporting metal layers. In case that the electrooptical (EO) layer is sufficiently thin, the SP's propagating along each of the metalEO layer boundaries are mutually coupled, resulting in two SP-system modes. For electrooptic modulation, coupling to that SP-system mode having the largest fractional power in the EO layer is utilized. The fundamental mode, launched into the silicon oxynitride $(\mathrm{SiON})$ waveguide at $z=0$, starts to couple to the strongly attenuated modes of the EO structure at $z=500 \mu \mathrm{m}$. The magnitude of this coupling is critically dependent on the phase-matching (PM) between the modes of the isolated waveguides. This PM can be modulated by a voltage across the EO polymer sandwiched between the two silver electrodes. This idea has been worked out to a laboratory sample of a SP-coupling based integrated optical intensity modulator. Its design, fabrication and characterization has been recently described in [5].

The behavior of the structure has been found to be very sensitive to changes of its parameters, so that accurate device modeling is a necessary prerequisite for its design. As the structure contains very thin and lossy metal layers supporting SP's, its accurate modeling is rather demanding.

These features stimulated the proposal to use it as another benchmark test for mutual comparison of various BPM's within the framework of the European Project COST 240. The benchmark test is defined as follows: calculate the throughput
TABLE I

Parameters of the EO Waveguide Structure Given in Fig. 1

\begin{tabular}{l|l|l}
\hline \multicolumn{1}{c|}{ layer } & \multicolumn{1}{|c|}{ index } & thickness $(\boldsymbol{\mu m})$ \\
\hline 1. air & 1.00 & semi-infinite \\
\hline 2. silver & $0.14-\mathrm{i} 11.0$ & 0.07 \\
\hline 3. polymer & $1.58-1.59$ & 1.1 \\
\hline 4. silver & $0.14-\mathrm{i} 11.0$ & 0.07 \\
\hline 5. $\mathrm{SiON}$ & 1.56 & 1.3 \\
\hline $6 . \mathrm{SiON}$ & 1.7 & 0.934 \\
\hline $7 . \mathrm{SiO}_{2}$ & 1.449 & semi-infinite \\
\hline
\end{tabular}

of the structure shown in Fig. 2 excited with its fundamental TM mode at the input at a wavelength of $\lambda=1.523 \mu \mathrm{m}$, for an EO polymer index $n_{p}$ varying in the region $1.58-1.59$. The materials, refractive indexes and thicknesses of the waveguide layers in the central part of the waveguide structure in Fig. 2 are given in Table I, counted from top to bottom.

The challenge to the numerical simulation methods arises from the high refractive-index contrast present in the structure and very different thicknesses of the layers, and it is interesting to see in what way the various methods (in particular the BPM methods) can cope with them.

In the next parts, the problem is analyzed, and results of the simulations are presented and discussed.

\section{ANALYSIS OF THE PROBLEM}

It is quite instructive to consider changes in the propagation constants, or effective mode indexes if isolated layer stacks are joined together. In Table II, the effective indexes of modes of various isolated layer stacks are given for $n_{p}=1.59$.

If we compare the mode indexes of $L_{1 \rightarrow 5}$ and $\mathrm{L}_{5 \rightarrow 7}$ with those of $L_{1 \rightarrow 7}$, considerable shifts are observed. This indicates a strong interaction between the two structures and a strong mixing of the original modal field of the SiON guide with guided modes, and probably also with radiation modes, of the EO guide. This can also be seen from the modal field solutions of the complete structure given in Fig. 3. This mixing, which can strongly be influenced by changing, e.g., the polymer index is the driving force for the attenuation of the launched fundamental TM mode. The left column of Fig. 3 shows the field profiles for the situation for $n_{p}=1.58685$ corresponding to strong mixing of the two lowest-order mode of $L_{1 \rightarrow 5}$ and $L_{5 \rightarrow 7}$ is shown in the right column. At the latter value of $n_{p}$, the effective indexes of these two modes are almost equal (1.62531-i.0.000617 for $L_{1 \rightarrow 5} ; 1.62444$ for $L_{5 \rightarrow 7}$ ) leading 
TABLE II

Effective Indexes of Lowest Order Guided Modes of Isolated Layer Stacks of the EO Modulator Defined in Fig. 1 and Table I. The Polymer Refractive Index Is $n_{p}=1.59$. The Short-Hand Notation $L_{i \rightarrow j}$ In the First Row Stands for a Structure Consisting of Layers $i$ to $j$ Inclusive, with the Outermost Layers Semi-Infinite

\begin{tabular}{c|c|c|c|c}
\hline $\begin{array}{c}\text { Mode } \\
\text { number }\end{array}$ & $L_{1 \rightarrow 3}$ & $L_{1 \rightarrow 5}$ & $L_{5 \rightarrow 7}$ & $L_{1 \rightarrow 7}$ \\
\hline 0 & $1.6070-\mathrm{i} 4.50 \times 10^{-4}$ & $1.6286-\mathrm{i} 6.18 \times 10^{-4}$ & $1.62444-\mathrm{i} 0$ & $1.6286-\mathrm{i} 6.15 \times 10^{-4}$ \\
\hline 1 & & $1.5761-\mathrm{i} 4.32 \times 10^{-4}$ & & $1.6253-\mathrm{i} 1.84 \times 10^{-5}$ \\
\hline 2 & & $1.5092-\mathrm{i} 9.91 \times 10^{-4}$ & & $1.5678-\mathrm{i} 5.23 \times 10^{-4}$ \\
\hline 3 & & & & $1.5061-\mathrm{i} 1.11 \times 10^{-3}$ \\
\hline
\end{tabular}
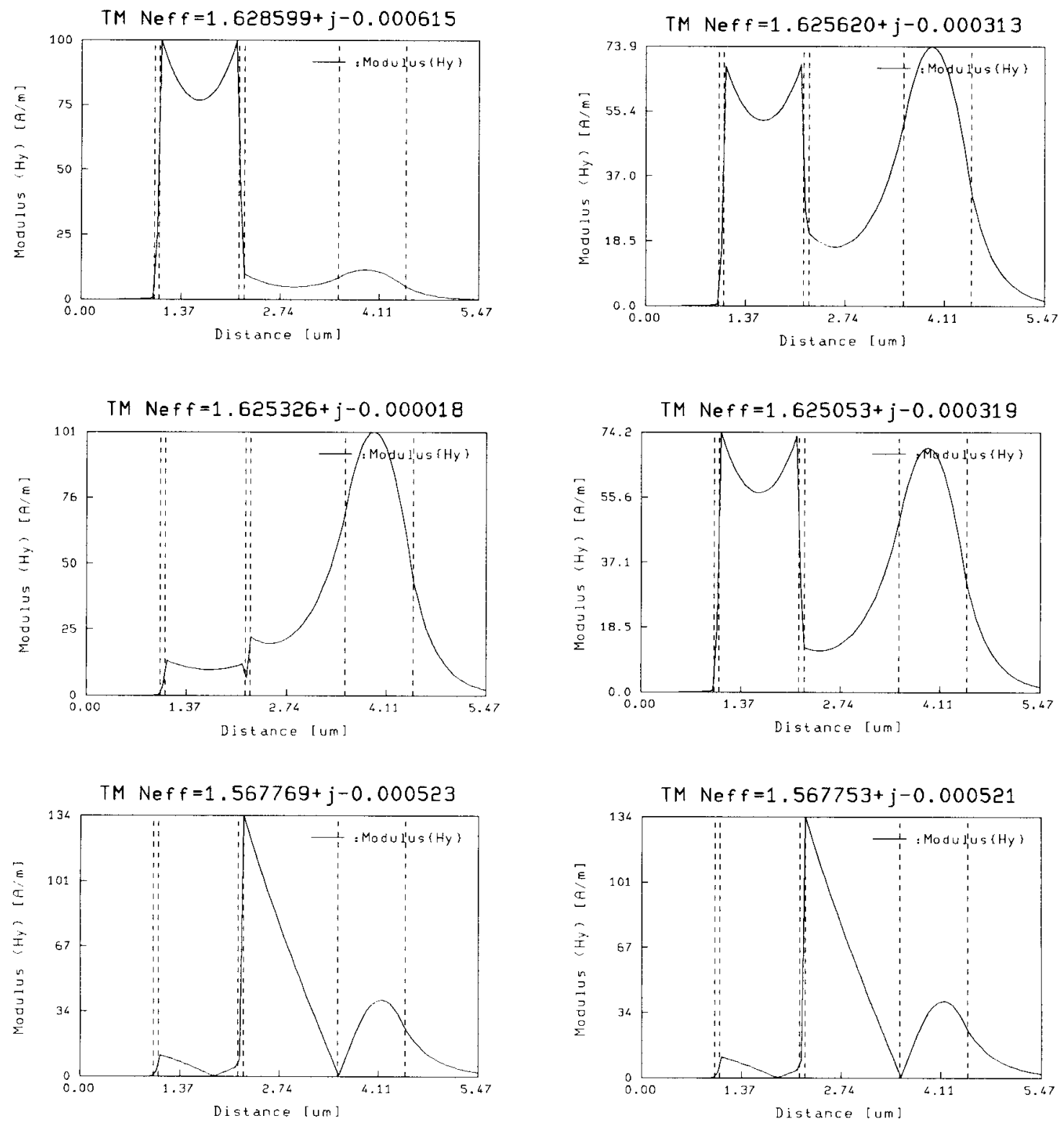

(a)

(b)

Fig. 3. The three lowest-order modal fields of the electrooptic waveguide structure for (a) $n_{p}=1.59$ and (b) $n_{p}=1.58685$.

to strong mixing in the joined structure. Note also, as can be seen from the modal fields at $N_{\text {eff }} \approx 1.5678$ in both Fig. 3, the (smaller) mixing between the first-order mode of $L_{1 \rightarrow 5}$ and the fundamental mode of $L_{5} \rightarrow 7$.

It might be clear from the discussion above that a simple coupled mode theory, involving only two modes, would not be sufficient for an accurate description of the behavior of light in the considered device. It can be seen from Fig. 3 (bottom pictures) that also power transfer to the (strongly damped) second-order $\left(L_{1 \rightarrow 7}\right.$ system) mode, at $z=500 \mu \mathrm{m}$, is not negligible, and also coupling to radiation modes might play a role. On the other hand, coupling back of these modes to 
TABLE III

Main Features of the Used Computational Methods. Abbreviations Used Here Are: IREE = Institute of Radio Engineering and Electronics, Prague, Czech Republic; FU Hagen = University for Distant Studies, Hagen; U Roma = University of Rome; U Twente = UnVersity of Twente; BEP = Bidirectional Eigenmode Propagation (Unidirectional Option) [6], [7]; MoL = Method of Lines [8]-[10]; FD2 BPM = Finite Difference Beam Propagation Method [11] Including a Second-Order Correction for the Slowly Varying Envelope Approximation (SVEA). If a NoneQuidistant Grid Spacing Is Used the SMALlest VALUE For this Is Given. EIC'S = EFFicient Interface Conditions [11]-[13]. "Sect." in the Stepsize Column Indicates That One Step Was Used For each $z$-Independent Section

\begin{tabular}{l|l|cc|l|l}
\hline Institute & Method & \multicolumn{2}{|c|}{$\begin{array}{c}\text { stepsize } \\
\Delta x / n m \Delta z / \mu m\end{array}$} & $\begin{array}{c}\text { interface } \\
\text { conditions }\end{array}$ & \multicolumn{1}{|c}{$\begin{array}{c}\text { Nr. of points/ } \\
\text { basis functions }\end{array}$} \\
\hline IREE & BEP & - & sect. & analytical & 16 modes \\
\hline FU Hagen & MoL & 25.9 & sect. & analytical/EIC's & equidistant \\
\hline U Roma & MoL & 1.0 & 10 & Fermi-function & $300 /$ non-equidistant \\
\hline U Twente & FD2 BPM & 2.0 & 1 & EIC's & $4000 /$ equidistant \\
\hline
\end{tabular}

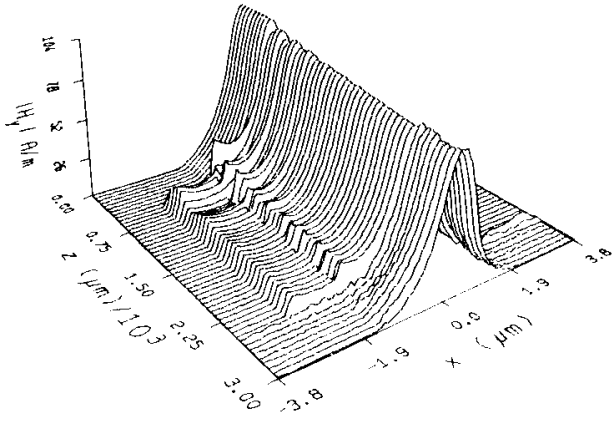

(a)

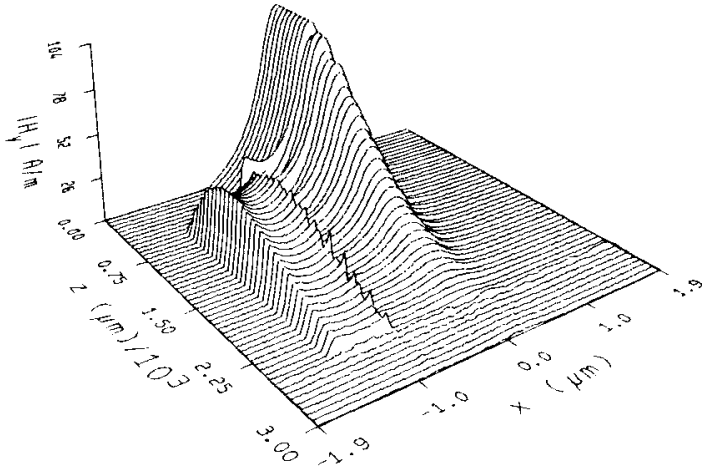

(b)

Fig. 4. Field propagation for two different polymer indexes: (a) $n_{p}=1.59$ and (b) picture $n_{p}=1.58685$.

waveguide $B$ at $z=2500 \mu \mathrm{m}$ is assumed to be negligible due to either the strong damping or the radiative character. This picture is confirmed by results of the various computational methods as argued below.

The computational methods should also be able to handle large index steps (note that $\Delta n^{2} \approx 120$ ) at the metal/dielectric interfaces. In particular, for methods based on a discretization in the transverse direction, an adequate treatment of the second-order derivative at these interfaces is crucial.

\section{The Applied Computational Methods}

All methods used here are well documented in the open literature (see Table III, and references given there) and will not be introduced here in full detail. The main features, as well as the most important computational parameters are given in Table III. All the presented methods, except the bidirectional method of lines (MoL) used by Fern Universität, Hagen, Germany, are unidirectional. The validity of the unidirectional approximation will be discussed next section.

Due to the large index contrast along the $x$-axis (transverse direction), all methods based on the discretization in that direction (i.e., MoL and finite difference (FD) BPM) needed special precautions for an accurate treatment of the secondorder derivative at these interfaces. Close to interfaces the effective interface conditions (EIC's) mentioned above use corrections in the standard three-point FD operator for the second-order derivative, which take into account the continuity of $H_{y}, \partial_{x} H_{y} / n^{2}$ and the discontinuity of $\partial_{x x} H_{y}$. More or less equivalent expressions for the corrected FD operator can be found in the literature [10]-[13]. Besides the EIC's for the dielectric media, the MoL of Fern Universität uses analytical functions in the metal layers. Due to these analytical expressions the field and its first derivative with respect to $\mathrm{x}$ were matched at the metal-dielectric interfaces giving a relation between the fields on the two sides of the metal layer. The finite difference scheme $(1-21)$ for these points was then replaced by expressions obtained by the analytical approach.

In the MoL of University of Rome the squared refractive index close to interfaces $\left(\varepsilon=n^{2}\right)$ is approximated by a Fermi function:

$$
\varepsilon_{\mathrm{Fermi}}=\varepsilon_{l}+\Delta \varepsilon /\{1+\exp (-\gamma x / \Delta x)\}
$$

Here $\Delta \varepsilon \equiv \varepsilon_{r}-\varepsilon_{1}$, the subscripts $r$ and $l$ denote right and left of the interface, receptively, $\Delta x$ is the stepsize and $\gamma$ is an adjustable parameter, maximizing the loss.

The average index used for the SVEA in the FD BPM was $n_{0}=1.624$, the window width was so large that reflection from the computational boundaries can be neglected.

The "unidirectional option" of BEP used by IREE means that multiple reflections from longitudinal discontinuities in the waveguide structure are neglected; their power was calculated to be very low, as discussed in the next section.

\section{RESUlTS AND DISCUSSION}

In Fig. 4 the field propagation is given, for two values of the polymer index, $n_{p}$, close to that for resonant coupling $\left(n_{p}=1.58685\right)$ and somewhat off resonance at $n_{p}=$ 1.59. The fields result from FD2 BPM calculations, the other 


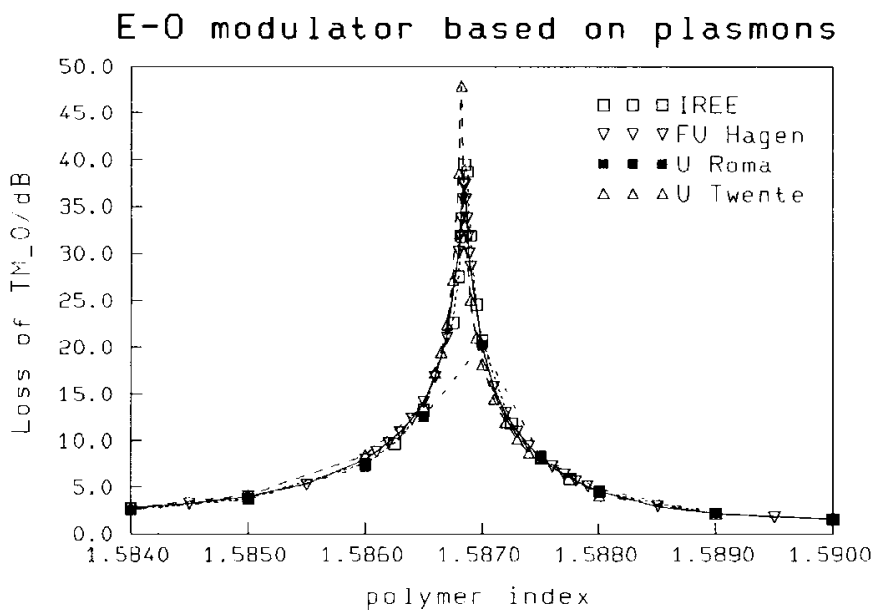

Fig. 5. Throughput loss as a function of the refractive index of the EO polymer layer.

methods give virtually identical results. From Fig. 4, it can be anticipated that (multiple) reflections might play a minor role. In particular, reflection from the end face of the EO structure is expected to be not completely negligible. A rough estimate, also taking into account that (in terms of local modes) only the strongly damped plasmon modes are partly reflected, leads to only a small error in the throughput of at most a few percent. This will influence the throughput, given in Fig. 5 not significantly. This is confirmed by comparison of the results from the bi- and unidirectional MoL by Fern Universität, which showed a difference of less than a tenth of a dB. Power reflection of the fundamental $\mathrm{TM}_{0}$ mode of the waveguide $\mathrm{B}$ reflected back from the EO section $(A+B$, see Fig. 2) was calculated by BEP to be below $-50 \mathrm{~dB}$.

Inspecting Fig. 5 it follows that the methods agree nicely and that the differences are small compared to accuracy's obtainable with most present fabrication technologies. A detail of the figure is given in Fig. 6. Here, the differences can be observed more clearly. They are attributed to discretization effects, investigated in more detail below. In order to understand the origin of these differences we remark that the loss is the result of a delicate interplay between modal indexes, both real and imaginary parts, and the overlap of the modal fields at the two transitions. We will first discuss this matter in more detail.

Using that the total field at transitions along the $z$-axis should be continuous and neglecting any reflection, the amplitude of the fundamental mode of waveguide $B$, at $z=$ $2500 \mu \mathrm{m}$, can be expressed as

$$
a_{0}^{B}(2500 \mu \mathrm{m})=a_{0}^{B}(500 \mu \mathrm{m}) \sum_{p} c_{0 p} \exp \left(-i \beta_{p} 2000 \mu \mathrm{m}\right)
$$

where

$$
\begin{aligned}
c_{0 p} \equiv & \int E_{x, 0}^{B} H_{y, p}^{\mathrm{EO}} \cdot d x \int E_{x, p}^{\mathrm{EO}} H_{y, 0}^{B} \\
& \cdot d x /\left(\int E_{x, 0}^{B} H_{y, 0}^{B} \cdot d x \int E_{x, p}^{\mathrm{EO}} H_{y, p}^{\mathrm{EO}} \cdot d x\right) \text { and } \\
& \sum_{p} c_{0 p}=1
\end{aligned}
$$

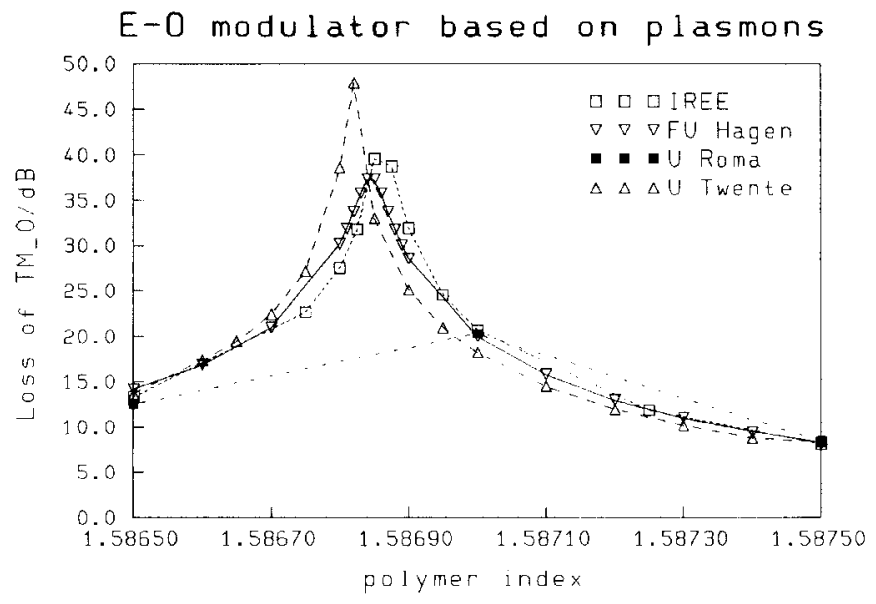

Fig. 6. Detail of Fig. 5 close to the peak.

should hold for a complete set of basis functions for the EO structure. Taking, for simplicity, into account only the two lower order modes of the EO structure $(p=0,1)$ it follows for the relative power at the output of the EO structure:

$$
\begin{aligned}
& \left|a_{0}^{B}(2500 \mu \mathrm{m}) / a_{0}^{B}(500 \mu \mathrm{m})\right|^{2} \\
& \quad=\left|c_{00}\right|^{2} \exp \left(-2 \beta_{0}^{\prime \prime} L\right)+\left|c_{01}\right|^{2} \exp \left(-2 \beta_{1}^{\prime \prime} L\right) \\
& \quad+2 \operatorname{Re}\left[c_{00} c_{01}^{*} \exp \left\{i\left(\beta_{1}^{*}-\beta_{0}\right) L\right\}\right] .
\end{aligned}
$$

Here, $L=2000 \mu \mathrm{m}$. Note that besides the two damping terms, the oscillatory term (last term above), representing the modal beat, may play an important role. In particular close to resonance this is the case for the EO structure. Using, for $n_{p}=1.58685$ the effective indexes given in Fig. 3 and the (approximate) coupling constants: $c_{00} 0.5501+i 0.522$ and $c_{01} 0.431-i 0.521$ it follows for the relative power:

$$
\begin{aligned}
& \left|a_{0}^{B}(2500 \mu \mathrm{m}) / a_{0}^{B}(500 \mu \mathrm{m})\right|^{2} \\
& \quad \approx 3.29 \times 10^{-3}-5.55 \times 10^{-3} \approx 1.0 e-4 \cong 40 \mathrm{~dB} .
\end{aligned}
$$

The three values above correspond to the three terms at the rhs of (5), and cancel each other almost completely, leading to the high loss of $40 \mathrm{~dB}$, close to the more exact values given in Fig. 6. So, as can be seen from (6) the loss depends very critically on the propagation constants and magnitude and complex phase of the transfer constants. For this reason the present benchmark test is a sensitive testing instrument for BPM's, in particular BPM's based on a discretization.

We have investigated the effect of the discretization by varying the lateral stepsize, $\Delta x$, in the FD BPM, with a value $n_{p}=1.58685$. Here, we have used both the standard method (i.e., without SVEA correction), denoted by FD0 BPM, and, as for Figs. 5 and 6 the FD2 BPM, see Fig. 7. As the discretization error in the effective index is proportional to $\Delta x^{2}$, the loss is given as a function of this quantity. It can be seen that the results converge to approximately the result of IREE, $39.54 \mathrm{~dB}$.

It can also be seen from Fig. 7 that the FD0 and FD2 BPM give almost identical values. This indicates that higher order 


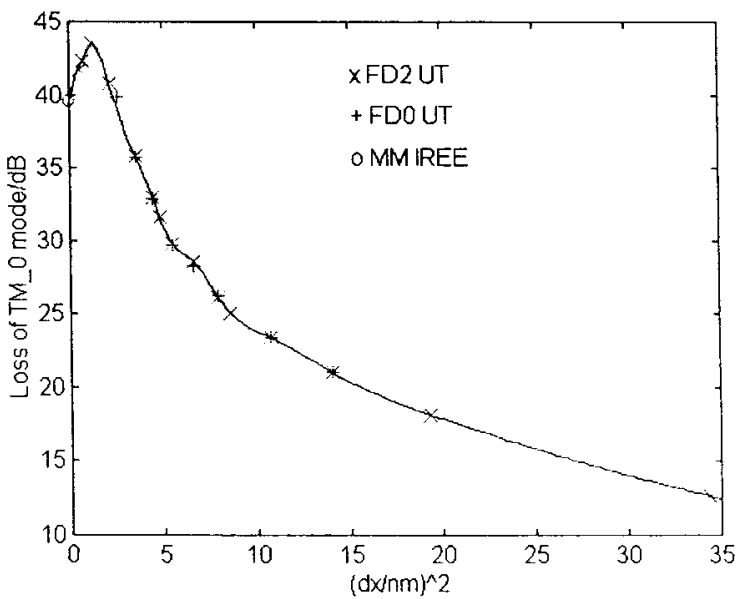

Fig. 7. Dependence of the loss in the test structure, calculated with the FD BPM, versus the square of the lateral stepsize $\Delta x^{2}$. The result of the BEP mode matching $(\mathrm{MM})$ is given for comparison.

modes and radiative modes, which are propagated with small phase-errors by the FD0 BPM, do not play an important role, i.e., these modes excited at the transition at $z=500 \mu \mathrm{m}$ will not reenter waveguide $B$ at $z=2500 \mu \mathrm{m}$, at least not very much. This picture is confirmed by BEP calculations which show a rapid convergence as a function of the number of basis functions.

So, as expected and also based on the above, we conclude that the BEP will give the (nearly) correct results for the device of the benchmark test. The other methods agree fairly well. The newly implemented analytical treatment of the high index layers, also using EIC's for the other layers, in the MoL by Fern Universität has also proven to be successful. This is probably also the case for the Fermi-function approach in the MoL by Universita di Roma, but more data around the resonance value of $n_{p}$ are required to be quite sure. As is well known, EIC's if applied in BPM's lead to a considerable improvement (or more rapid convergence). Nevertheless, for structures like the present one, it would be advantageous to limit the computation time by further improvements. For example, by introduction of a EIC's for a five-point FD operator, if possible converging proportional to $\Delta x^{4}$.

\section{CONCLUSIONS}

We have performed a benchmark test using a waveguide structure of an electrooptic modulator, comparing the method of lines, mode matching, and the finite difference beam propagation method. By comparing the results, also varying the parameters for the computations we conclude [about the device for the test (Fig. 2)]:

- for switching index changes of $10^{-3}$ are required;

- the transmission loss is less than $3 \mathrm{~dB}$;

- the extinction is $-40 \mathrm{~dB}$, and better than $-30 \mathrm{~dB}$ within an index range of $\Delta n \approx 10^{-4}$;

- the calculated loss depends in a very critical way on the interplay between propagation constants, both real and imaginary parts, and the modal overlap at transitions in the structure;
- the large index contrast at metal-dielectric interfaces require a careful interface treatment at these interfaces for methods based on a discretization.

and about the applied methods (see Table II, Figs. 5-7):

- the results of the applied methods agree fairly well, e.g., differences in the calculated loss peak position correspond to a change of less than $10^{-4}$ in the EO-polymer index;

- small differences are attributed to discretization errors;

- newly introduced methods for the treatment of the finite difference operator near interfaces, and the field in highindex layers appear to perform well;

- the BEP seems to be most suitable for the test structure;

- for computational schemes based on a discretization, further acceleration of the convergence, as a function of lateral stepsize, would be desirable for structures with large index contrast as in the structure for the benchmark test.

\section{REFERENCES}

[1] H.-P. Nolting and R. März, "Results of benchmark tests for different numerical BPM algorithms," J. Lightwave Technol., vol. 13, pp. 216-224, 1995.

[2] J. Haes et al., "A comparison between different propagative schemes for the simulation of tapered step index slab waveguides," J. Lightwave Technol., vol. 14, pp. 1557-1567, 1996.

[3] H.-P.Nolting, G. Sztefka, M. Grawert, and J.Ctyroky, "Wave propagation in the waveguide with a balance of gain and loss," Integrated Photon. Res., Boston, MA, Tech. Dig. Series, Apr. 29-May 2, 1996, vol. 6, pp. 76-79.

[4] H. J. M. Kreuwel, P. V. Lambeck, J. M. M. Beltman, and Th. J. A. Popma, "Mode coupling in multi-layered structures applied to a chemical sensor and a wavelength selective directional coupler," in Proc. ECIO, 1987, pp. 217-222.

[5] A. Driessen, H. M. M. K. Koerkamp, and Th. J. A. Popma, "Novel integrated optic intensity modulator based on mode coupling," Fiber Integrated Opt., vol. 13, pp. 445-461, 1994.

[6] G. Sztefka and H.-P. Nolting, "Bidirectional eigenmode propagation for large refractive index steps," IEEE Photon. Technol. Lett., vol. 5, pp. 554-557, 1993.

[7] J Ctyroký, J. Homola, and M. Skalský, "Modeling of surface plasmon resonance waveguide sensor by complex mode expansion and propagation method," Opt. Quantum Electron., vol. 29, pp. 301-311, 1997.

[8] J. Gerdes and R. Pregla, "Beam-propagation algorithm based on the method of lines," J. Opt. Soc. Amer. B, vol. 8, no. 2, pp. 389-394, 1991.

[9] R. Pregla, "MoL-BPM method of lines based beam propagation method," in Methods for Modeling and Simulation of Guided-Wave Optoelectronic Devices, W. P. Huang, Ed., number PIER 11, and also in Progress in Electromagnetic Research. Cambridge, MA: EMW Publishing, 1995, pp. 51-102.

[10] S. Helfert and R. Pregla, "Finite difference expressions for arbitrarily positioned dielectric steps in waveguide structures," J. Lightwave Technol., vol. 14 , pp. 2414-2421, Oct. 1996.

[11] H. J. W. M. Hoekstra, "On beam propagation methods for modeling in integrated optics," Opt. Quantum Electron., vol. 29, pp. 157-171, 1997.

[12] C. Vassallo, "Improvement of finite difference methods for step-index optical waveguides," Inst. Elect. Eng. Proc. J., vol. 139, pp. 137-142, 1992.

[13] H. J. W. M. Hoekstra, G. J. M. Krijnen, and P. V. Lambeck, " Efficient interface conditions for the finite difference beam propagation method," J. Lightwave Technol., vol. 10, pp. 1352-1355, 1992.

H. J. W. M. Hoekstra, photograph and biography not available at the time of publication. 
P. V. Lambeck, photograph and biography not available at the time of publication.

G. J. M. Krijnen, photograph and biography not available at the time of publication.

J. Ctyroky, photograph and biography not available at the time of publication.

M. De Minicis, photograph and biography not available at the time of publication.
C. Sibilia, photograph and biography not available at the time of publication.

O. Conradi, photograph and biography not available at the time of publication.

S. Helfert, for a biography, see p. 1702 of the September 1998 issue of this JOURNAL.

R. Pregla (M'76-SM'83), for a biography, see p. 1702 of the September 1998 issue of this JOURNAL. 